\title{
Practical implementation of noninvasive ventilation in Amyotrophic Lateral Sclerosis: lessons learned from a clinical case series
}

\author{
Elizabeth C. Parsons, MD, MSc ${ }^{1}$, John C. Carter, $\mathrm{MD}^{2}$, Joanna E. Wrede, $\mathrm{MD}^{3}$, Lucas M. Donovan, $\mathrm{MD}^{4}$, Brian N. Palen, $\mathrm{MD}^{1}$
}

EC Parsons, JC Carter, JE Wrede, LM Donovan, BN Palen. Practical implementation of noninvasive ventilation in Amyotrophic Lateral Sclerosis: lessons learned from a clinical case series. Can J Respir Ther 2019;55:13-15. doi: 10.29390/cjrt-2018-020.

\begin{abstract}
Purpose: Noninvasive ventilation (NIV) may improve survival and quality of life in Amyotrophic Lateral Sclerosis (ALS) patients. There is a surprising paucity of practical guidelines for office-based implementation and management of NIV outside of tertiary ALS centers. We saw the need for a clinical protocol to allow feasible and consistent NIV management in this patient population.

Methods: We created a clinical protocol for office-based initiation of NIV implemented on consecutive ALS patients referred from our regional ALS multidisciplinary clinic. The protocol provided initial empiric settings using a bilevel device in volume-assured pressure support mode. A respiratory therapist (RT) initiated NIV in an office setting and made adjustments according to patient tolerance and therapy targets outlined in the protocol. Later setting changes were performed at patient or provider request. We evaluated patient adherence and efficacy via device download at 30 days and 1 year.

Results: We present data from a case series of the first 14 consecutive patients initiated on NIV over a 20-month period. Our protocol underwent iterative modification based on clinical experience and patient feedback. Early challenges included the significant time and resource burden required to coordinate device downloads and patient follow-up. Early 30-day NIV adherence was variable (median 20 out of 30 days), while 1-year NIV adherence was excellent (median 27.5 out of 30 days)

Conclusions: Our RT-driven clinical NIV protocol was feasible but labor intensive. Achieving real-world adherence of NIV in our ALS patients required iterative protocol adjustment, significant RT provider time, and tele-based follow-up.
\end{abstract}

Key Words: Amyotrophic Lateral Sclerosis; motor neuron disease; noninvasive ventilation; hypoventilation; quality improvement

\section{INTRODUCTION}

Amyotrophic Lateral Sclerosis (ALS) is a progressive neuromuscular degenerative disease that culminates in respiratory failure and death [1]. Noninvasive ventilation (NIV) improves quality of life and long-term survival in ALS [2,3]. Unfortunately, NIV initiation and maintenance are often hampered by this patient population's high clinical care needs and rapidly evolving physiology including progressive reductions in chest wall compliance, compromised ability to trigger breaths, bulbar dysfunction [4], and poor upper extremity dexterity.

Clinicians caring for ALS patients are faced with controversial data on clinical indications for NIV initiation [5] and a paucity of practical guidelines for NIV setup and long-term management. Clinical care is further hampered by the population's evolving physiologic characteristics requiring frequent device and interface adjustments [6-8] as well as the concentration of experienced specialists at a handful of tertiary care centers.

We provide pulmonary consultation services for the multidisciplinary ALS program at the VA Puget Sound, a referral hub for veterans across the Pacific Northwest. We recognized the need for and created an officebased protocol for NIV initiation and follow-up in the ALS population. Our hope was that a practical protocol would enable consistent NIV care at our own facility as well as enhance care continuity for veterans sharing care at partner facilities without experienced ALS providers.

In this paper, we present a case series of the first 14 consecutive ALS patients initiated on NIV using our office-based protocol. We hope that sharing our results will open a dialogue on practical aspects of NIV therapy in ALS and improve consistency of care.

\section{METHODS}

\section{Clinical Setting \& Initiation Criteria}

The multidisciplinary ALS program at VA Puget Sound Health Care System serves an average panel of 90 ALS patients from a large geographic catchment area including three states in the Pacific NorthwestWashington, Idaho, and Alaska. This program is led by Rehabilitation Medicine with consultation available from the Departments of Pulmonary Medicine and Respiratory Therapy. Consecutive ALS patients were referred to Pulmonary for NIV evaluation/education based on institution protocol including any of the following criteria: orthopnea; forced vital capacity (FVC) less than $70 \%$ predicted; sniff nasal inspiratory force $<40 \mathrm{~cm} \mathrm{H}_{2} \mathrm{O}$ or maximal inspiratory pressure $\leq 60 \mathrm{~cm} \mathrm{H}_{2} \mathrm{O}$; provider judgement based on symptoms including

\footnotetext{
${ }^{1}$ Division of Pulmonary, Critical Care and Sleep Medicine, Veterans Affairs Puget Sound Health Care System, University of Washington, Seattle, WA

${ }^{2}$ Division of Pulmonary, Critical Care, and Sleep Medicine, Case Western Reserve University School of Medicine and MetroHealth, Cleveland, OH

${ }^{3}$ Division of Pulmonary and Sleep Medicine and Division of Neurology, Seattle Children's Hospital, Seattle, WA

${ }^{4}$ Division of Pulmonary, Critical Care and Sleep Medicine, University of Washington, Seattle, WA

Correspondence: Lucas M. Donovan, MD, University of Washington, Division of Pulmonary, Critical Care, and Sleep Medicine, 1959 NE Pacific Street, Seattle, WA 98105. Tel.: (206) 543-3166; E-mail: ldonovan@u.washington.edu
}

Published online at https://www.cjrt.ca on 09 January 2019

OPEN ACCESS

This open-access article is distributed under the terms of the Creative Commons Attribution Non-Commercial License (CC BY-NC) (http:// creativecommons.org/licenses/by-nc/4.0/), which permits reuse, distribution and reproduction of the article, provided that the original work is properly cited and the reuse is restricted to noncommercial purposes. For commercial reuse, contact editor@csrt.com 
progressive dyspnea, headaches, daytime somnolence, frequent nocturnal awakenings; or patient desire to initiate therapy.

\section{NIV PROTOCOL}

The NIV protocol offered stepwise guidance for NIV initiation criteria, setup, and adjustment using patient feedback and a targeted assessment of ventilation (protocol provided in Supplementary data ${ }^{1}$ ). For this case series, we began therapy utilizing a NIV device with the volume-assured pressure support mode, iVAPS (ResMed ${ }^{\mathrm{TM}}$ ). We initially based our noninvasive ventilation settings on manufacturer's recommendations for their volume-assured pressure support (VAPS) mode. As we will discuss in further detail in the results section, some of our patients were intolerant of these settings, and we modified the original protocol to less aggressive pressure and volume targets. We also modified the protocol to include NIV modes.

\section{NIV INITIATION AND MANAGEMENT LOGISTICS}

Our consultative care was provided via a respiratory therapist (RT) supervised by a pulmonary physician with ALS expertise. NIV was initiated during an outpatient clinic visit (scheduled for 90 minutes). Initially these visits were performed with both the pulmonologist and RT. Later after a 3-month period of training, the RT implemented the protocol alone. The patient was oriented to the device and mask interface using initial settings aimed at a target tidal volume and minute ventilation. Device settings were further adjusted to achieve target ventilation goals and comfort/tolerance with the patient awake and recumbent.

NIV follow-up was limited by travel burden in this population. Patients were provided with the direct phone number of the RT so they could initiate telephone or in-person follow-up at any time. Patients in our ALS multidisciplinary clinic undergo rigorous, full-day evaluations led by Rehabilitation Medicine every 3 months. NIV follow-up with the RT was available (but not mandatory) at provider or patient request at each 3-month multidisciplinary visit. Therapy was adjusted per protocol based on patient-reported symptoms and information from data downloads; no routine labs were obtained. Remote-enabled devices were not yet available at our facility, thus device downloads were performed manually (in person or via mail).

\section{Protocol Evaluation and Adjustment}

A total of 14 consecutive ALS patients initiated NIV using our protocol between January 2014 and October 2015. This quality-improvement initiative was conducted under the auspices of the VA Office of Specialty Care and the Office of Rural Health. In accordance with VHA Handbook 1058.05, we obtained approval of nonresearch status from the VA program office and did not require institutional review board review. For this type of study, formal consent is not required. All clinical care was provided in accordance with the ethical standards of our usual clinical care and in accordance with the 1964 Helsinki declaration and its later amendments. The protocol underwent multiple adjustments to meet the clinical needs of our patients.

\section{Data Analysis}

We present descriptive statistics as mean with standard deviation or median with interquartile range (IQR) depending on the normality of distribution. We assessed correlations using Spearman's correlation as many of our measures were not normally distributed. For correlations, we used a $p \leq 0.05$ as the threshold for significance.

\section{RESULTS}

\section{Patient Characteristics and NIV Adherence}

Patients presented for NIV setup at a mean of 2.9 years following ALS diagnosis, with a mean maximum inspired pressure of $-61 \mathrm{~cm} \mathrm{H}_{2} \mathrm{O}$, median FVC of $60 \%$ predicted, and median ALS Functional Rating

\footnotetext{
${ }^{1}$ Supplementary data available online at https://www.cjrt.ca/doi10-29390cjrt-2018-020suppl/
}

Scale (ALSFRS) of 29 (Table 1). Only four patients had previously been on positive airway pressure therapy.

At 30 days after setup, 13 patients remained on NIV (one patient switched to continuous positive airway pressure for intolerance) (Table 2). NIV usage by download was highly variable and did not correlate with either FVC (Spearman's $\rho=0.30, p=0.32$ ) or ALSFRS score (Spearman's $\rho=0.10, p=0.75$ ).

At one year after setup, nine patients remained on NIV (four patients died). Download data were available for six patients. In a 30-day period, these patients used NIV for a median of 27.5 days (IQR 26-30 days) for a median of 606 minutes (IQR 191-641 minutes) usage per night.

\section{Feedback and Protocol Modification}

At early stages of protocol implementation, our initial standard mode of ventilation was VAPS with a target minute ventilation of $6-8 \mathrm{~mL} / \mathrm{kg}$. However, many patients complained of excessive pressure, and download data also showed delivered ventilation exceeding targeted values (Table 2). We adjusted the protocol to begin therapy at a lower target ventilation of $4 \mathrm{~mL} / \mathrm{kg}$. We also initially used the standard auto-adjusting backup rate. We found that approximately one-third of patients experienced dyssynchrony which improved with the use of a lower set respiratory rate. Ultimately, we arrived at a set rate of 2 breaths less than the observed respiratory rate in clinic. Finally, in a subset of patients who found the fluctuations in pressure delivered by VAPS to be uncomfortable, we would transition from VAPS to bilevel with or without a backup rate (see Supplementary data ${ }^{1}$ ).

Most patients preferred to follow-up and address concerns via phone with our RT. Our reliance on manual or mail-in downloads posed challenges in obtaining downloads and making pressure adjustments in a timely fashion. Unfortunately, devices with remote download/adjustment capability were not yet available at our institution.

\section{TABLE 1}

\section{Patient characteristics at NIV setup $(n=14)$}

\begin{tabular}{lc}
\hline Age in years, mean (SD) & $66.5(8.6)$ \\
Years since ALS symptom onset, median (IQR) & $2(1-3)$ \\
ALS Functional Rating Score, median (IQR) & \\
(range 0 worst to 40 best) & $29(23-32)$ \\
FVC (\% predicted), median (IQR) & \\
FVC less than 50\% predicted, $n(\%)$ & $60(40-70)$ \\
MIP in $\mathrm{cm} \mathrm{H}_{2} \mathrm{O}$, mean (SD) & $6(43)$ \\
MEP in $\mathrm{cm} \mathrm{H} \mathrm{H}_{2} \mathrm{O}$, median (IQR) & $61(23)$ \\
Previously on PAP therapy, $n(\%)$ & $65(56-84)$ \\
\hline
\end{tabular}

Note: NIV, noninvasive ventilation; ALS, Amyotrophic Lateral Sclerosis; IQR, interquartile range; FVC, forced vital capacity; MIP, maximum inspiratory pressure; MEP, maximum expiratory pressure; $P A P$, positive airway pressure. ${ }^{*}$ Available for $n=8$

${ }^{\dagger}$ Closest values to time of setup

\section{TABLE 2}

NIV adherence and efficacy by 30-day download $(n=13)$

\begin{tabular}{ll}
\hline Days used, median (IQR) & $20(8-29)$ \\
Days with $\geq 4-\mathrm{h}$ use, median (IQR) & $2(0-21)$ \\
Average hours on days used, median (IQR) & $1.8(1.3-7.2)$ \\
Usage of $\geq 4$ h per night on $\geq 70 \%$ of nights, $n(\%)$ & $4(31 \%)$ \\
Leak, median (IQR) & $5.5(1.2-16)$ \\
AHI, median (IQR) & $14.8(1.9,24.8)$ \\
Median TV delivered in mL/kg, mean (SD) & $6.7(2.0)$ \\
Median RR, mean (SD) & $15.8(3)$ \\
Median MV in L/min, mean (SD) & $8.3(2.3)$
\end{tabular}

Note: NIV, noninvasive ventilation; IQR, interquartile range; $A H I$, apnea hypopnea index; $T V$, tidal volume; $R R$, respiratory rate; $M V$, minute ventilation. 


\section{DISCUSSION}

Early evaluation of our office-based NIV protocol for ALS demonstrated that it was feasible yet resource intensive. We encountered clinical, logistic, and operational challenges. Modifying our protocol to less aggressive initial ventilation targets and incorporating telemedicine strategies for follow-up proved valuable in improving NIV care for our patients. Our findings highlight the unique challenges of NIV adherence and follow-up in ALS, an issue that receives little attention in publications on this topic. When we performed a PubMed search of these terms we found no published trials relating to interventions designed to improve adherence in this population.

The main clinical challenge encountered was early intolerance. In a clinical case series, we cannot determine if the improved NIV usage among 1-year survivors was a result of modifying our protocol to less aggressive ventilation targets, clinical progression, or other unmeasured factors. Early NIV usage did not appear to correlate with standard disease markers (ALSFRS, functional residual capacity), consistent with previously published data [7-10]. While attractive due to their frequent availability, these measures are in fact non-specific for hypoventilation. Future modification of our protocol will incorporate clinical triggers that may be more specific to hypoventilation, including daytime hypoxia or hypercapnia, headaches, sleep disturbances, daytime somnolence, and positional dyspnea [11].

Additionally, our project encountered logistical and infrastructure challenges. Obtaining manual downloads and face-to-face follow-up were difficult for patients, especially those with advanced disease. Remoteenabled devices (not available at the time of our project) are crucial in caring for patients with ALS on NIV, and we have since implemented these as a standard practice. Telephone, online, and telemedicine strategies may also be needed. Lastly, our program required a full-time, dedicated RT. NIV setups and troubleshooting for ALS patients are time consuming even for an experienced RT, sometimes requiring consultation with a supervising physician.

Our clinical experience with NIV in ALS should be interpreted within the context of several important limitations. Our office-based protocol was designed for clinical care; it was not a research project designed to rigorously assess clinical effectiveness or patient outcomes. Our choice of NIV device was based on availability at our institution and does not reflect a preference or recommendation of a specific device or mode. Choice of NIV mode is complicated by an abundance of technologies and limited comparative data. Our experience suggests that mode is less important than iterative adjustment to patient needs and systematically addressing adherence barriers.

Our clinical protocol is neither a definitive nor an exhaustive answer to all the challenges of NIV care in ALS. We continue to refine our protocol to meet the specific needs of our ALS patients, and we believe that standardized protocols may certainly be useful in the leveraging of ALS-specific resources across partner institutions. By sharing our clinical experience, we hope to highlight important practical considerations for the clinician interested in NIV therapy in ALS.

\section{ACKNOWLEDGEMENTS}

The authors would like to acknowledge Rolando Nicolas, RRT, who performed device setup, clinical follow-up, and data downloads.

\section{CONFLICT OF INTEREST}

All authors certify that they have no affiliations with or involvement in any organization or entity with any financial interest (such as honoraria; educational grants; participation in speakers' bureaus; membership, employment, consultancies, stock ownership, or other equity interest; and expert testimony or patent-licensing arrangements), or non-financial interest (such as personal or professional relationships, affiliations, knowledge or beliefs) in the subject matter or materials discussed in this manuscript.

\section{FUNDING}

Lucas Donovan is supported by salary support from the National Institutes of Health (NIH), T32HL007287-38, F32 HL140685-01 and receives additional grant funding from the American Thoracic Society ASPIRE (Academic Sleep Pulmonary Integrated REsearch/Clinical) Fellowship. No other funding was provided for this work. The sponsor had no role in the design or conduct of this research. Furthermore, this project was performed as part of routine clinical care, under the auspices of quality improvement, at the VA Puget Sound Medical Center. Contents do not represent the views of the US. Department of Veterans Affairs or the United States Government.

\section{REFERENCES}

1. Albdewi MA, Liistro G, El Tahry R. Sleep-disordered breathing in patients with neuromuscular disease. Sleep Breath. 2017 Jul 12. doi: 10.1007/ s11325-017-1538-x

2. Radunovic A, Annane D, Rafig MK, Brassington R, Mustfa N. Mechanical ventilation for amyotrophic lateral sclerosis / motor neuron disease. Cochrane Database Syst Rev. 2017 Oct 6;10:CD004427. doi: 10.1002/14651858.CD004427.pub4

3. Bourke SC, Tomlinson M, Williams TL, Bullock RE, Shaw PJ. Gibson GJ. Effects of non-invasive ventilation on survival and quality of life in patients with amyotrophic lateral sclerosis: A randomised controlled trial. Lancet Neurol. 2006 Feb;5(2):140-7. doi:10.1016/S1474-4422(05) 70326-4

4. Veldhuis SK, Doff MH, Stegenga B, Nieuwenhuis JA, Wijkstra PJ. Oral appliance to assist non-invasive ventilation in a patient with amyotrophic lateral sclerosis. Sleep Breath. 2015 Mar;19(1):61-3. doi: 10.1007/ s11325-014-1021-x

5. Miller RG, Jackson CE, Kasarskis EJ, et al. Practice parameter update: The care of the patient with amyotrophic lateral sclerosis: Drug, nutritional, and respiratory therapies (an evidence-based review): Report of the Quality Standards Subcommittee of the American Academy of Neurology. Neurology. 2009 Oct 13;73(15):1218-26. doi: 10.1212/ WNL.0b013e3181bc0141

6. Berlowitz DJ, Howard ME, Fiore JF, Jr., et al. Identifying who will benefit from non-invasive ventilation in amyotrophic lateral sclerosis/motor neurone disease in a clinical cohort. J Neurol Neurosurg Psychiatry 2016 Mar;87(3):280-6. doi: 10.1136/jnnp-2014-310055

7. Jackson CE, Rosenfeld J, Moore DH, et al. A preliminary evaluation of a prospective study of pulmonary function studies and symptoms of hypoventilation in ALS/MND patients. J Neurol Sci. 2001 Oct 15;191 $(1-2): 75-8$.

8. Czaplinski A, Yen AA, Appel SH. Forced vital capacity (FVC) as an indicator of survival and disease progression in an ALS clinic population. J Neurol Neurosurg Psychiatry. 2006 Mar;77(3):390-2. doi: 10.1136/ jnnp.2005.072660

9. Lechtzin N, Scott Y, Busse AM, Clawson LL, Kimball R, Wiener CM. Early use of non-invasive ventilation prolongs survival in subjects with ALS. Amyotroph Lateral Scler 2007;8(3):185-8. doi: 10.1080/ 17482960701262392

10. Carratu P, Spicuzza L, Cassano A, et al. Early treatment with noninvasive positive pressure ventilation prolongs survival in Amyotrophic Lateral Sclerosis patients with nocturnal respiratory insufficiency. Orphanet J Rare Dis. 2009 Mar 10;4:10. doi: 10.1186/1750-1172-4-10

11. Piper AJ, Yee BJ. Hypoventilation syndromes. Compr Physiol. 2014 Oct;4(4): 1639-76. doi: 10.1002/cphy.c140008 